\title{
GENETIX
}

\section{The QArray series: new answers for protein arrays}

Screening tens to hundreds of proteins for enzymatic activity or protein interactions can now be performed in a quantitative and economical manner using protein microarrays. Recent validated examples include assays for human protein kinases and protein interactions with p53. Here we describe the QArray series of instruments for the printing and assaying of such arrays. Of particular note is printing of arrays into microwell plates, a methodology that combines the benefits of arrays and the convenience of a conventional assay format.

Genetix markets a number of products for protein arraying. All three arrayers, QArrayMini, QArray2 or QArrayMax use pins for spotting proteins onto surfaces (Fig. 1). These give less than $3 \%$ variation under standard conditions, and work to date indicates that the spotted proteins maintain their activity and are not denatured or inactivated by contact with the tungsten pins or various array surfaces. In the early development of protein arrays, much controversy surrounded whether the arrays should be made by contact printing with pins or by noncontact methods. Pins are now accepted as the industry standard. The difficulty with noncontact systems is that accumulation of static charge can cause inaccurate deposition of the spots (Fig. 2), and the expensive and delicate arraying pipettes have to be cleaned between the application of each protein to an array. In the case when many different proteins are arrayed on a relatively small number of slides $(<100)$, this adds substantially to the time required to make the arrays.

Arraying DNA onto surfaces usually requires either modification of the surface to create a three-dimensional surface or chemical modification (amide, aldehyde or epoxy groups) to couple the oligonucleotides or cDNAs to the array substrate.

Protein arrays are created by printing spots of protein (for example, antibodies, recombinant proteins, peptides) on a solid surface such as a glass slide coated with a protein-binding chemistry. For such arrays, it is typical to modify the surface with molecules such as nickel or streptavidin to bind His-tagged or biotin-labeled proteins, respectively. This, of course, limits the range of proteins to be arrayed and requires specific surfaces for specific protein con-

Julian F Burke ${ }^{1}$, Cheryl Munro² \& Chris J Mann

${ }^{1}$ Genetix Limited, New Milton, Hampshire, BH255NN UK. ${ }^{2}$ Genetix Inc., 56 Roland Street, Suite 106, Boston, Massachusetts 02129, USA. Correspondence should be addressed to J.F.B. (julian.burke@genetix.com).

PUBLISHED ONLINE 20 APRIL 2006; DOI:10.1038/NMETH878

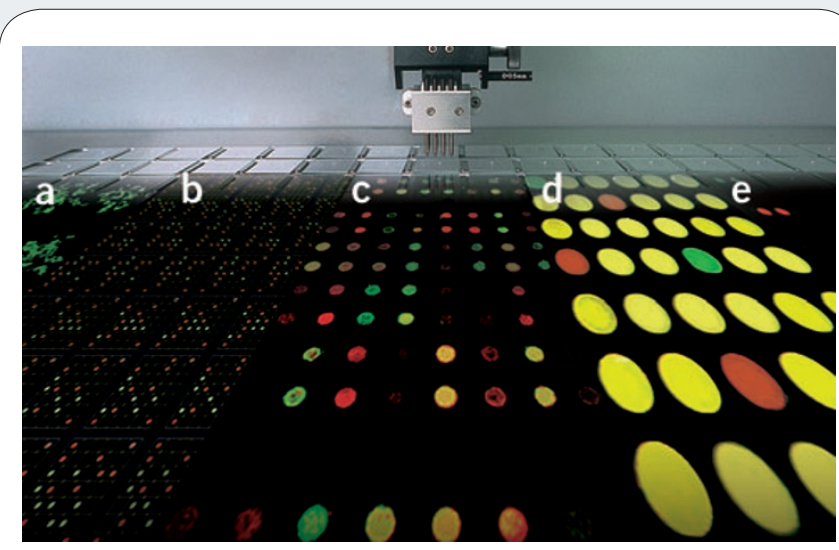

Figure 1 | Spot morphology produced by Genetix microarrayers arraying a variety of biological molecules (artificial colors). (a) Reverse transfection array showing expression of a fluorescent protein. (b) Arrays of proteins in a 96microwell plate. (c) cDNA array hybridized with Cy3- and Cy5-labeled probe. (d) Array of Cy3-labeled human lgG. (e) Comparative genomic hybridization (Cy3 and Cy5) of arrayed BAC

structs. The production of proteins in bacterial or human cell culture systems are well-established procedures, and such proteins can be expressed with specific fusion tags for immobilization to appropriately coated solid surfaces. For nontagged proteins, the same surfaces used for DNA arrays such as amine, aldehyde or epoxy chemistries can be used for the capture of proteins onto the array.

Two factors that are important for arraying proteins are humidity and temperature. These can be regulated in the Genetix QArray series of arrayers.

Examples of proteins that have been successfully arrayed on chips are kinases and p53 (ref. 1). These are clearly important in cell signaling, and validate the approach of using arrays for investigating both the substrate specificity of mutants (DNA binding for p53 using a fluorescent labeled oligonucleotide) and substrate specificity ${ }^{2,3}$. 
APPLICATION NOTES

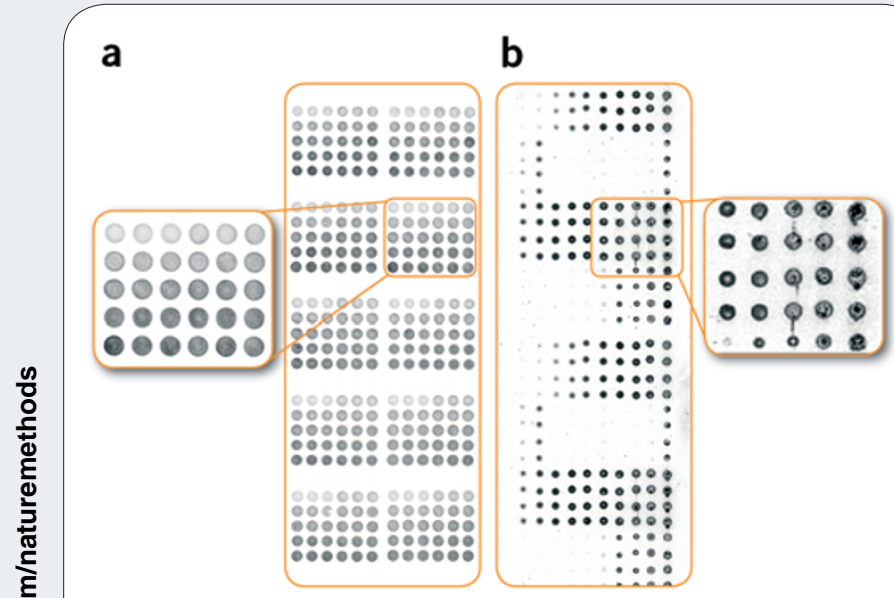

Figure $\mathbf{2}$ | Comparison of protein arrays produced by a Genetix microarrayer and a commercial noncontact printer. (a) Genetix arrayer spotting Cy3-labeled human lgG. (b) A commercial noncontact microarrayer spotting labeled protein (not identified)

As these are signaling molecules, the protein interactions can also be measured on these arrays.

The screening of serum for antigens, using protein arrays requires much smaller sample volumes compared to a standard microwell format. The amount of serum required to develop an array containing over 10,000 features can be the same as that required to develop a single feature in a well of a 96-well plate. Genetix microarrayers have been used for a range of such applications ${ }^{4-8}$. There are some cases, however, in which microwells have an advantage in that they can be easier to handle with existing instrumentation. For example, the Genetix QArray ${ }^{M i n i}$ and QArray 2 have been optimized to generate high-quality protein arrays both in individual wells of a microwell plate and on slides on the same instrument. These can be printed at a density of up to 100 spots per well in a 96-well plate, and up to 16 spots in a 384-well plate (Fig. 3). This method is particularly useful in diagnostic or clinical applications in which many samples are tested in parallel against only a few specific proteins of interest. The hybrid bed on the Genetix arrayers allows both slides and plates to be printed on the same instrument.

Other important advances in this field include the development of techniques for studying carbohydrates on proteins ${ }^{9}$ using lectins. This is an area of increasing importance for diagnostics. Finally,

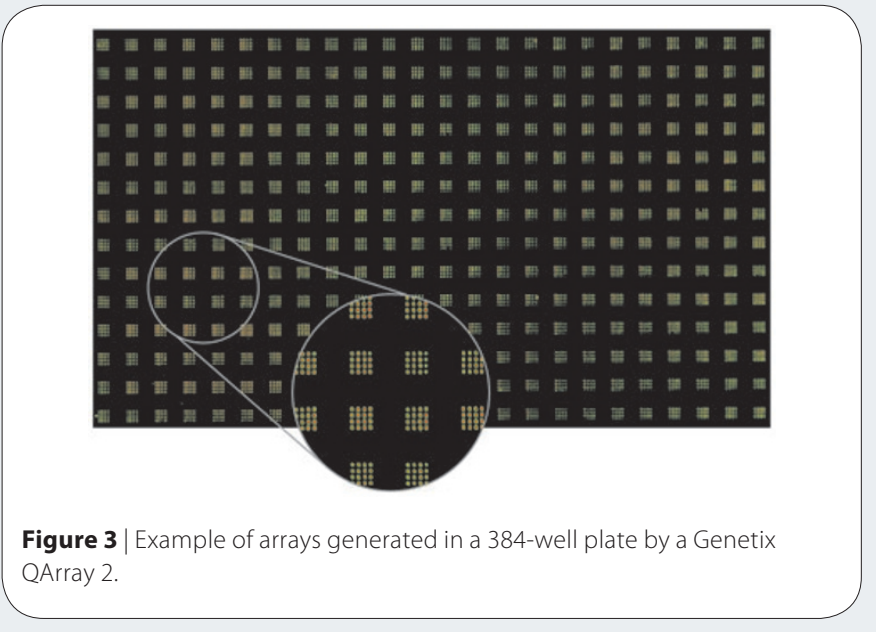

arraying proteins allows screening of metabolites and proteins that bind them ${ }^{10}$. This is a real challenge, as small metabolites cannot easily be labeled, and the future for such studies may lie in arrays in which the interaction is subsequently detected by Raman or mass spectroscopy.

1. Boutell, J.M. et al. Functional protein microarrays for parallel characterisation of p53 mutants. Proteomics 4, 1950-1958 (2004).

2. Kramer, A. et al. Identification of barley CK2 targets by using protein microarray technology. Phytochemistry 65, 1777-1784 (2004).

3. Feilner, T. et al. High-throughput identification of potential Arabidopsis mitogen-activated protein kinases substrates. Mol. Cell. Proteomics 10, 1558-68 (2005).

4. Lueking, A. et al. A nonredundant human protein chip for antibody screening and serum profiling. Mol. Cell. Proteomics 2, 1342-1349 (2003).

5. Gutjahr, C. et al. Mouse protein arrays from a TH1 cell cDNA library for antibody screening and serum profiling. Genomics 85, 285-296 (2005).

6. Angenendt, P. et al. Next generation of protein microarray support materials: evaluation for protein and antibody microarray applications. $J$. Chromatogr. A 1009, 97-104 (2003).

7. Angenendt, P. et al. 3D protein microarrays: performing multiplex immunoassays on a single chip. Anal. Chem. 75, 4368-4372 (2003).

8. Kersten, B. et al. Generation of Arabidopsis protein chips for antibody and serum screening. Plant Mol. Biol. 52, 999-1010 (2003).

9. Feizi, T. \& Chai, W. Oligosaccharide microarrays to decipher the glyco code. Nat. Rev. Mol. Cell. Biol. 5, 582-588 (2004).

10. Griffin, J.L. et al. An integrated reverse functional genomic and metabolic approach to understanding orotic acid-induced fatty liver. Physiol. Genomics 17, 140-149 (2004).

This article was submitted to Nature Methods by a commercial organization and has not been peer reviewed. Nature Methods takes no responsibility for the accuracy or otherwise of the information provided. 